hep-ph/9505327

FTUV/95-21

IFIC/95-21

May 1995

\title{
Constraining new interactions with leptonic tau decays
}

\author{
Antonio Pich and João P. Silva \\ Departament de Física Teòrica and IFIC \\ Universitat de València - CSIC \\ Dr. Moliner 50, E-46100 Burjassot, València, Spain
}

\begin{abstract}
The recent measurements of the Michel parameters in tau decays enable, for the first time, a thorough analysis of the leptonic sector. In general, in models beyond the Standard Model, these parameters will be altered through changes in the $\mathrm{W}$ and $\mathrm{Z}$ couplings, and/or through interactions mediated by new gauge bosons. We perform a complete, model independent analysis of the constraints imposed by the present data on such boson-mediated interactions, and point out the existence of useful relations among the couplings.
\end{abstract}

\section{Introduction}

In any theory in which the fermions have interactions mediated by heavy (scalar and/or vector) bosons, the low-energy consequences can be conveniently parametrized by fourfermion interactions. Hence, precise low-energy tests of processes involving fermions constitute a window through which one may peek into the nature of the interactions at higher energies. This procedure is, in principle, cleaner in the lepton sector where it is not obscured by hadronization. Furthermore, one usually expects the scalar-mediated interactions to have the fermionic vertices proportional to the fermion masses, thus making the tau decays the ideal system for their study.

The exciting new experimental results in leptonic tau decays reported recently [1, 2, 3] provide most of the missing pieces of information and warrant for the first time a complete analysis of the lepton sector.

In the Standard Model (SM), the quantum numbers of the fermions under $\mathrm{SU}(2)_{L}$ are judiciously chosen in order to obtain a low-energy " $(\mathrm{V}-\mathrm{A}) \otimes(\mathrm{V}-\mathrm{A})$ " four-fermion structure, correctly describing the dominant features of the experiments in $\beta$ and $\mu$ decays. One has now the opportunity to test this scheme in tau decays. Should any difference arise, that will be a sign of Physics Beyond the SM. In most extensions of the 
SM, these new effects arise through differences in the couplings to the $W$ and $Z$ bosons, or through the exchange of new intermediate bosons. The new four-fermion interactions thus obtained will be typically dominated by a single intermediate boson; either the one with the smallest mass or that whose couplings to the leptons are specially large. In any case, important relations exist between the low energy parameters.

This program is undertaken in what follows. In section 2 we set up the analysis in terms of the helicity projection form of the four-fermion interaction pointing out the most salient model-independent features. We do this for completeness and to set up the notation for the subsequent sections. In section 3 we summarize the experimental situation and in section 4 we discuss the universality tests. Section 5 is devoted to the analysis of non-standard charged intermediate bosons and section 6 to lepton-flavourchanging neutral-boson interactions. Section 7 contains a summary of some features of our analysis and resulting information on the opportunities for physics beyond the SM. Finally, we draw our conclusions in section 8. The appendix is devoted to the development of relations relevant for the analysis of lepton-flavour-changing neutral bosons and a detailed discussion of the consequences of the unobservability of the finalstate neutrinos. We also discuss there the complementary information extractable from neutrinoless charged-lepton decays.

\section{The four-fermion hamiltonian}

Let us consider the leptonic decays $l^{-} \rightarrow \nu_{l} l^{\prime-} \bar{\nu}_{l^{\prime}}$, where the lepton pair $\left(l, l^{\prime}\right)$ may be $(\mu, e),(\tau, e)$, or $(\tau, \mu)$. The most general derivative-free, lepton-number conserving, four-lepton interaction hamiltonian, consistent with locality and Lorentz invariance, can be written as $[4$

$$
\mathcal{H}=4 \frac{G_{l^{\prime} l}}{\sqrt{2}} \sum_{\epsilon, \omega=R, L}^{n=S, V, T} g_{l_{\epsilon}^{\prime} l_{\omega}}^{n}\left[\overline{l_{\epsilon}^{\prime}} \Gamma^{n}\left(\nu_{l^{\prime}}\right)_{\sigma}\right]\left[\overline{\left(\nu_{l}\right)_{\lambda}} \Gamma_{n} l_{\omega}\right]
$$

The label $n$ refers to the type of interaction, namely

$$
\Gamma^{S}=1, \quad \Gamma^{V}=\gamma^{\mu}, \quad \Gamma^{T}=\frac{1}{\sqrt{2}} \sigma^{\mu \nu} \equiv \frac{i}{2 \sqrt{2}}\left(\gamma^{\mu} \gamma^{\nu}-\gamma^{\nu} \gamma^{\mu}\right)
$$

for the scalar, vector and tensor interactions, respectively. The neutrino chiralities, $\sigma$ and $\lambda$, are uniquely determined once $n$ and the charged-lepton chiralities, $\epsilon$ and $\omega$, are chosen. Thus, one has 19 real constants, since there are only two non-zero tensor terms and one global phase may be taken away.

In any reasonable model, these couplings are the low-energy limit of scalar and/or vector-boson mediated transitions. In general, several such contributions will exist and we write

$$
g_{l_{\epsilon}^{\prime} l_{\omega}}^{n}=w_{l_{\epsilon}^{\prime} l_{\omega}}^{n}+a_{l_{\epsilon}^{\prime} l_{\omega}}^{n}+b_{l_{\epsilon}^{\prime} l_{\omega}}^{n}+\cdots
$$

where the letter $w$ is reserved for the known $W$ boson, and each letter $(a, b, \ldots)$ refers to couplings originating from a given additional intermediate boson. From $W$ decays, as well as from $\beta$ and $\mu$ decays, we know that the $W$ vertices with leptons will 
necessarily give the dominant contribution to the $\tau$ and $\mu$ leptonic decays. The SM predicts that this is the only contribution, and, moreover, that there are only couplings to left-handed leptons. Hence, in the SM

$$
g_{l_{L}^{\prime} l_{L}}^{V} \equiv w_{l_{L}^{\prime} l_{L}}^{V}=1
$$

and all other couplings are predicted to vanish. Of course, what one measures in these decays are the sum $g_{l_{\epsilon}^{\prime} l_{\omega}}^{n}$ of all the different contributions with the same chiral structure and these may interfere constructively or destructively.

For an initial lepton-polarization $\mathcal{P}_{l}$, the final charged lepton distribution in the decaying lepton rest frame is usually parametrized in the form [5, 6]

$$
\begin{aligned}
\frac{d^{2} \Gamma(x, \cos \theta)}{d x d \cos \theta}= & \frac{m_{l} \omega^{4}}{2 \pi^{3}}\left(G_{l^{\prime} l}^{2} N\right) \sqrt{x^{2}-x_{0}^{2}}\left\{x(1-x)+\frac{2}{9} \rho\left(4 x^{2}-3 x-x_{0}^{2}\right)+\eta x_{0}(1-x)\right. \\
& \left.-\frac{1}{3} \mathcal{P}_{l} \xi \sqrt{x^{2}-x_{0}^{2}} \cos \theta\left[1-x+\frac{2}{3} \delta\left(4 x-4+\sqrt{1-x_{0}^{2}}\right)\right]\right\}
\end{aligned}
$$

where $\theta$ is the angle between the $l^{-}$spin and the final charged-lepton momentum, $\omega \equiv\left(m_{l}^{2}+m_{l^{\prime}}^{2}\right) / 2 m_{l}$ is the maximum $l^{\prime-}$ energy for massless neutrinos, $x \equiv E_{l^{\prime}} / \omega$ is the reduced energy and $x_{0} \equiv m_{l^{\prime}} / \omega$. For unpolarized $l^{\prime} s$, the distribution is characterized by the so-called Michel [7] parameter $\rho$ and the low-energy parameter $\eta$. Two more parameters, $\xi$ and $\delta$ can be determined when the initial lepton polarization is known. If the polarization of the final charged lepton is also measured, 5 additional independent parameters [8] $\left(\xi^{\prime}, \xi^{\prime \prime}, \eta^{\prime \prime}, \alpha^{\prime}, \beta^{\prime}\right)$ appear.

To determine the constraints on Physics Beyond the SM, it is convenient to express the Michel parameters in terms of their deviation from the SM values [9]. One obtains,

$$
\begin{gathered}
\rho-\frac{3}{4}=-\frac{3}{4 N}\left[\left|g_{L R}^{V}\right|^{2}+\left|g_{R L}^{V}\right|^{2}+2\left|g_{L R}^{T}\right|^{2}+2\left|g_{R L}^{T}\right|^{2}+\operatorname{Re}\left(g_{L R}^{S} g_{L R}^{T *}+g_{R L}^{S} g_{R L}^{T *}\right)\right] \\
\eta=\frac{1}{2 N} \operatorname{Re}\left[g_{L L}^{S} g_{R R}^{V *}+g_{L R}^{S} g_{R L}^{V *}+g_{R L}^{S} g_{L R}^{V *}+g_{R R}^{S} g_{L L}^{V *}+6\left(g_{L R}^{V} g_{R L}^{T *}+g_{R L}^{V} g_{L R}^{T *}\right)\right] \\
\xi-1=-\frac{1}{2 N}\left[\left|g_{L R}^{S}\right|^{2}+\left|g_{R R}^{S}\right|^{2}+4\left(-\left|g_{L R}^{V}\right|^{2}+2\left|g_{R L}^{V}\right|^{2}+\left|g_{R R}^{V}\right|^{2}\right)\right. \\
\left.-4\left|g_{L R}^{T}\right|^{2}+16\left|g_{R L}^{T}\right|^{2}-8 \operatorname{Re}\left(g_{L R}^{S} g_{L R}^{T *}-g_{R L}^{S} g_{R L}^{T *}\right)\right] \\
(\xi \delta)-\frac{3}{4}=-\frac{3}{4 N}\left[\frac{1}{2}\left(\left|g_{L R}^{S}\right|^{2}+\left|g_{R R}^{S}\right|^{2}\right)+\left(\left|g_{L R}^{V}\right|^{2}+\left|g_{R L}^{V}\right|^{2}+2\left|g_{R R}^{V}\right|^{2}\right)\right. \\
\left.+2\left(2\left|g_{L R}^{T}\right|^{2}+\left|g_{R L}^{T}\right|^{2}\right)-\operatorname{Re}\left(g_{L R}^{S} g_{L R}^{T *}-g_{R L}^{S} g_{R L}^{T *}\right)\right] .
\end{gathered}
$$

We set the overall normalization factor

$$
\begin{aligned}
N \equiv & \frac{1}{4}\left(\left|g_{L L}^{S}\right|^{2}+\left|g_{L R}^{S}\right|^{2}+\left|g_{R L}^{S}\right|^{2}+\left|g_{R R}^{S}\right|^{2}\right)+\left(\left|g_{L L}^{V}\right|^{2}+\left|g_{L R}^{V}\right|^{2}+\left|g_{R L}^{V}\right|^{2}+\left|g_{R R}^{V}\right|^{2}\right) \\
& +3\left(\left|g_{L R}^{T}\right|^{2}+\left|g_{R L}^{T}\right|^{2}\right),
\end{aligned}
$$

to 1 , as it is frequently done. This may always be donet by absorbing it in the definition

${ }^{1}$ Alternatively, one may absorb $N$ for the $(\mu, e)$ pair, say, use a common $G_{F}$ in Eq. If and keep the normalization factor $N$ for the other two decays. However, care must then be taken when using published bounds for the coupling constants $g_{l_{\epsilon}^{\prime} l_{\omega}}^{n}$, since the normalization in Eq. (7) is usually adopted. 
of $G_{l^{\prime} l}^{2}$. We note that the parameters $\eta$ and $G_{l^{\prime} l}^{2}$ are the only ones linear in the newphysics contributions. Namely, they have terms proportional to

$$
\eta \sim \frac{1}{2} \operatorname{Re}\left(1 \times g_{R R}^{S *}\right)
$$

and

$$
G_{l^{\prime} l}^{2} \propto 1+2 \operatorname{Re}\left(1 \times \Delta g_{L L}^{V *}\right),
$$

where we have used the fact that the SM contribution to $g_{L L}^{V}$ is approximately 1 , and new contributions to $g_{L L}^{V}$ have been parametrized by $\Delta g_{L L}^{V}$. Clearly this last type of variation is only detectable if it is non-universal.

It is convenient to introduce [10] the probabilities $Q_{\epsilon \omega}$ for the decay of a $\omega$-handed $l^{-}$into an $\epsilon$-handed daughter lepton,

$$
\begin{aligned}
Q_{L L}=\frac{1}{4}\left|g_{L L}^{S}\right|^{2}+\left|g_{L L}^{V}\right|^{2} & =\frac{1}{4}\left(-3+\frac{16}{3} \rho-\frac{1}{3} \xi+\frac{16}{9} \xi \delta+\xi^{\prime}+\xi^{\prime \prime}\right), \\
Q_{R R}=\frac{1}{4}\left|g_{R R}^{S}\right|^{2}+\left|g_{R R}^{V}\right|^{2} & =\frac{1}{4}\left(-3+\frac{16}{3} \rho+\frac{1}{3} \xi-\frac{16}{9} \xi \delta-\xi^{\prime}+\xi^{\prime \prime}\right), \\
Q_{L R}=\frac{1}{4}\left|g_{L R}^{S}\right|^{2}+\left|g_{L R}^{V}\right|^{2}+3\left|g_{L R}^{T}\right|^{2} & =\frac{1}{4}\left(5-\frac{16}{3} \rho+\frac{1}{3} \xi-\frac{16}{9} \xi \delta+\xi^{\prime}-\xi^{\prime \prime}\right), \\
Q_{R L}=\frac{1}{4}\left|g_{R L}^{S}\right|^{2}+\left|g_{R L}^{V}\right|^{2}+3\left|g_{R L}^{T}\right|^{2} & =\frac{1}{4}\left(5-\frac{16}{3} \rho-\frac{1}{3} \xi+\frac{16}{9} \xi \delta-\xi^{\prime}-\xi^{\prime \prime}\right) .
\end{aligned}
$$

Upper bounds on any of these (positive-semidefinite) probabilities translate into corresponding limits for all couplings with the given chiralities.

The total decay rate is given by

$$
\Gamma=\frac{m_{l}^{5} G_{l^{\prime} l}^{2}}{192 \pi^{3}}\left\{f\left(\frac{m_{l^{\prime}}^{2}}{m_{l}^{2}}\right)+4 \eta \frac{m_{l^{\prime}}}{m_{l}} g\left(\frac{m_{l^{\prime}}^{2}}{m_{l}^{2}}\right)\right\} r_{\mathrm{RC}},
$$

where

$$
\begin{aligned}
& f(z)=1-8 z+8 z^{3}-z^{4}-12 z^{2} \ln z \\
& g(z)=1+9 z-9 z^{2}-z^{3}+6 z(1+z) \ln z
\end{aligned}
$$

Thus, the normalization $G_{e \mu}$ corresponds to the Fermi coupling $G_{F}$, measured in $\mu$ decay.

The factor

$$
r_{\mathrm{RC}}=\left[1+\frac{\alpha\left(m_{l}\right)}{2 \pi}\left(\frac{25}{4}-\pi^{2}\right)\right]\left[1+\frac{3}{5} \frac{m_{l}^{2}}{M_{W}^{2}}-2 \frac{m_{l^{\prime}}^{2}}{M_{W}^{2}}\right],
$$

takes into account radiative corrections not included in the Fermi coupling constant $G_{F}$, and the non-local structure of the $W$ propagator. These effects [1] are quite small: $r_{\mathrm{RC}}^{\tau \rightarrow \mu, e}=0.9960 ; r_{\mathrm{RC}}^{\mu \rightarrow e}=0.9958$. Notice, that the we are adopting the usual procedure of taking the radiative corrections within the Standard Model. Since we assume that the Standard Model provides the dominant contribution to the decay rate, any additional 
higher-order correction beyond the effective four-fermion Hamiltonian (11) would be a subleading effect.

The kinematical integrations have been done assuming massless neutrinos. The numerical correction induced by a non-zero $\nu_{l}$ mass, $r_{\nu_{l}} \equiv 1+\delta_{\nu_{l}} \approx 1-8\left(m_{\nu_{l}} / m_{l}\right)^{2}$, is quite small. The present experimental upper limits [8, 12] on the neutrino masses imply: $\left|\delta_{\nu_{\mu}}^{\mu \rightarrow e}\right|<5 \times 10^{-5}(90 \% \mathrm{CL}),\left|\delta_{\nu_{\mu}}^{\tau \rightarrow \mu}\right|<2 \times 10^{-7}(90 \% \mathrm{CL}),\left|\delta_{\nu_{\tau}}^{\tau \rightarrow \mu, e}\right|<1.4 \times 10^{-3}$ (95\% CL).

It is fortunate that the two parameters which are linear in the new-physics contributions, $\eta$ and $G_{l^{\prime} l}^{2}$, are precisely the ones which survive in the total decay width. One can then study them with non-universality searches which already provide very precise tests of the lepton sector.

\section{$3 \quad$ Experimental summary}

For $\mu$-decay, where precise measurements of the polarizations of both $\mu$ and $e$ have been performed, there exist [10] upper bounds on $Q_{R R}, Q_{L R}$ and $Q_{R L}$, and a lower bound on $Q_{L L}$. They imply corresponding upper bounds on the 8 couplings $\left|g_{R R}^{n}\right|,\left|g_{L R}^{n}\right|$ and $\left|g_{R L}^{n}\right|$. The measurements of the $\mu^{-}$and the $e^{-}$do not allow us to determine $\left|g_{L L}^{S}\right|$ and $\left|g_{L L}^{V}\right|$ separately [10, 13]. Nevertheless, since the helicity of the $\nu_{\mu}$ in pion decay is experimentally known to be -1 , a lower limit on $\left|g_{L L}^{V}\right|$ is obtained 10 from the inverse muon decay $\nu_{\mu} e^{-} \rightarrow \mu^{-} \nu_{e}$. The present (90\% CL) bounds 80 on the $\mu$-decay couplings are given in Table 1. These limits show nicely that the bulk of the $\mu$-decay transition amplitude is indeed of the predicted V-A type.

\begin{tabular}{|c|c|c|}
\hline$\left|g_{e_{L} \mu_{L}}^{S}\right|<0.55$ & $\left|g_{e_{L} \mu_{L}}^{V}\right|>0.96$ & - \\
$\left|g_{e_{R} \mu_{R}}^{S}\right|<0.066$ & $\left|g_{e_{R} \mu_{R}}^{V}\right|<0.033$ & - \\
$\left|g_{e_{L} \mu_{R}}^{S}\right|<0.125$ & $\left|g_{e_{L} \mu_{R}}^{V}\right|<0.060$ & $\left|g_{e_{L} \mu_{R}}^{T}\right|<0.036$ \\
$\left|g_{e_{R} \mu_{L}}^{S}\right|<0.424$ & $\left|g_{e_{R} \mu_{L}}^{V}\right|<0.110$ & $\left|g_{e_{R} \mu_{L}}^{T}\right|<0.122$ \\
\hline
\end{tabular}

Table 1: 90\% CL experimental limits [8] for the $\mu$-decay $g_{e_{\epsilon} \mu_{\omega}}^{n}$ couplings.

The experimental analysis of the $\tau$-decay parameters is necessarily different from the one applied to the muon, because of the much shorter $\tau$ lifetime. The measurement of the $\tau$ polarization and the parameters $\xi$ and $\delta$ is still possible due to the fact that the spins of the $\tau^{+} \tau^{-}$pair produced in $e^{+} e^{-}$annihilation are strongly correlated [14-23]. However, the polarization of the charged lepton emitted in the $\tau$ decay has never been measured. In principle, this could be done for the decay $\tau^{-} \rightarrow \mu^{-} \bar{\nu}_{\mu} \nu_{\tau}$ by stopping the muons and detecting their decay products [20]. The measurement of the inverse decay $\nu_{\tau} l^{-} \rightarrow \tau^{-} \nu_{l}$ looks far out of reach.

The present experimental status on the $\tau$-decay Michel parameters is shown in Table 2, which gives the world-averages of all published [1, 2, 3, 8] measurements. The improved accuracy of the most recent experimental analyses has brought an enhanced sensitivity to the different shape parameters, allowing the first measurements of $\eta_{\tau \rightarrow \mu}$ [1], 2], $\xi_{\tau \rightarrow e}, \xi_{\tau \rightarrow \mu},(\xi \delta)_{\tau \rightarrow e}$ and $(\xi \delta)_{\tau \rightarrow \mu}$ [四]. (The ARGUS measurement [3] of $\xi_{\tau \rightarrow l}$ and 
$(\xi \delta)_{\tau \rightarrow l}$ assumes identical couplings for $l=e, \mu$. A measurement of $\sqrt{\xi_{\tau \rightarrow e} \xi_{\tau \rightarrow \mu}}$ was published previously [24]).

\begin{tabular}{|c|c|c|c|c|}
\hline Parameter & $\tau^{-} \rightarrow \mu^{-}$ & $\tau^{-} \rightarrow e^{-}$ & With Lepton-Universality & SM \\
\hline$\rho$ & $0.738 \pm 0.038$ & $0.736 \pm 0.028$ & $0.733 \pm 0.022$ & 0.75 \\
$\eta$ & $-0.14 \pm 0.23$ & - & $-0.01 \pm 0.14$ & 0 \\
$\xi$ & $1.23 \pm 0.24$ & $1.03 \pm 0.25$ & $1.06 \pm 0.11$ & 1 \\
$\xi \delta$ & $0.71 \pm 0.15$ & $1.11 \pm 0.18$ & $0.76 \pm 0.09$ & 0.75 \\
\hline
\end{tabular}

Table 2: Experimental averages of the $\tau$-decay Michel parameters [1, 2, 3, 8]. The fourth column assumes lepton universality.

The determination of the $\tau$-polarization parameters [1, 3, 25], allows us to bound the total probability for the decay of a right-handed $\tau$,

$$
Q_{\tau_{R}} \equiv Q_{l_{R}^{\prime} \tau_{R}}+Q_{l_{L}^{\prime} \tau_{R}}=\frac{1}{2}\left[1+\frac{\xi}{3}-\frac{16}{9}(\xi \delta)\right] .
$$

One finds (ignoring possible correlations among the measurements):

$$
\begin{aligned}
& Q_{\tau_{R}}^{\tau \rightarrow \mu}=0.07 \pm 0.14<0.28 \\
& Q_{\tau_{R}}^{\tau \rightarrow e}=-0.32 \pm 0.17<0.14 \quad(90 \% \mathrm{CL}), \\
& Q_{\tau_{R}}^{\tau \rightarrow l}=0.00 \pm 0.08<0.14 \quad(90 \% \mathrm{CL}),
\end{aligned}
$$

where the last value refers to the $\tau$-decay into either $l=e$ or $\mu$, assuming universal leptonic couplings. Since these probabilities are positive semidefinite quantities, they imply corresponding limits on all $\left|g_{l_{R} \tau_{R}}^{n}\right|$ and $\left|g_{l_{L} \tau_{R}}^{n}\right|$ couplings. The quoted 90\% CL have been obtained adopting a Bayesian approach for one-sided limits [8]. Table 3] gives the implied bounds on the $\tau$-decay couplings.

\begin{tabular}{||c|c||c||}
\hline$\tau \rightarrow \mu$ & $\tau \rightarrow e$ & $\tau \rightarrow l$ \\
\hline \hline$\left|g_{\mu_{R} \tau_{R}}^{S}\right|<1.05$ & $\left|g_{e_{R} \tau_{R}}^{S}\right|<0.75^{*}$ & $\left|g_{l_{R} \tau_{R}}^{S}\right|<0.74$ \\
$\left|g_{\mu_{L} \tau_{R}}^{S}\right|<1.05$ & $\left|g_{e_{L} \tau_{R}}^{S}\right|<0.75^{*}$ & $\left|g_{l_{L} \tau_{R}}^{S}\right|<0.74$ \\
\hline$\left|g_{\mu_{R} \tau_{R}}^{V}\right|<0.53$ & $\left|g_{e_{R} \tau_{R}}^{V}\right|<0.38^{*}$ & $\left|g_{l_{R} \tau_{R}}^{V}\right|<0.37$ \\
$\left|g_{\mu_{L} \tau_{R}}^{V}\right|<0.53$ & $\left|g_{e_{L} \tau_{R}}^{V}\right|<0.38^{*}$ & $\left|g_{l_{L} \tau_{R}}^{V}\right|<0.37$ \\
\hline$\left|g_{\mu_{L} \tau_{R}}^{T}\right|<0.30$ & $\left|g_{e_{L} \tau_{R}}^{T}\right|<0.22^{*}$ & $\left|g_{l_{L} \tau_{R}}^{T}\right|<0.21$ \\
\hline
\end{tabular}

Table 3: $90 \%$ CL limits for the $\tau_{R}$-decay $g_{l_{\epsilon} \tau_{R}}^{n}$ couplings. The numbers with an asterisk use the measured value of $(\xi \delta)_{e}$; the meaning of the assigned confidence level could be doubtful in this case (see text).

Notice, however, that the central value of $Q_{\tau_{R}}^{\tau \rightarrow e}$ turns out to be negative at the $2 \sigma$ level; i.e. , there is only a $3 \%$ probability to have a positive value of $Q_{\tau_{R}}^{\tau \rightarrow e}$. Therefore, the limits on $\left|g_{e_{R} \tau_{R}}^{n}\right|$ and $\left|g_{e_{L} \tau_{R}}^{n}\right|$ should be taken with some caution, since the meaning of the assigned confidence level is not at all clear. 
The problem clearly comes from the measured value of $(\xi \delta)_{e}$. In order to get a positive probability $Q_{\tau_{R}}$, one needs $(\xi-1)>\frac{16}{3}\left[(\xi \delta)-\frac{3}{4}\right]$. Thus, $(\xi \delta)$ can only be made larger than $3 / 4$ at the expense of making $\xi$ correspondingly much larger than one. Hence, if the current values of the Michel parameters for the decay of tau into electron and neutrinos were to be confirmed, one would have to go beyond the effective hamiltonian of Eq. (1D): the combined observations for $\xi_{\tau \rightarrow e}$ and $(\xi \delta)_{\tau \rightarrow e}$ are not consistent with an effective four-fermion interaction of the form in Eq. (1). That is to say that no flavour-conserving, derivative-free, four-lepton interaction can be found, satisfying both these results simultaneously. Further, since lepton-flavour violations have no measurable effect if the final neutrinos are massless and unobserved [26], and derivative couplings would be suppressed by $m_{\tau}^{2} / M_{W}^{2} \sim 5 \times 10^{-4}$, a sizeable effect not included in the effective Hamiltonian (1) seems very unlikely ${ }^{2}$. Hence, based solely on theoretical grounds, one can achieve the conclusion that, within a four-fermion hamiltonian, either $(\xi \delta)_{\tau \rightarrow e}$ comes into agreement with the SM, or $\xi_{\tau \rightarrow e}$ must move by a factor close to 2 (a most unreasonable proposition).

Table $⿴$ q gives the world-average values of $m_{\tau}, \tau_{\tau}, B_{l} \equiv \operatorname{Br}\left(\tau^{-} \rightarrow \nu_{\tau} l^{-} \bar{\nu}_{l}\right), B_{\pi} \equiv$ $\operatorname{Br}\left(\tau^{-} \rightarrow \nu_{\tau} \pi^{-}\right), B_{K} \equiv \operatorname{Br}\left(\tau^{-} \rightarrow \nu_{\tau} K^{-}\right)$and $B_{h} \equiv \operatorname{Br}\left(\tau^{-} \rightarrow \nu_{\tau} \pi^{-}+\nu_{\tau} K^{-}\right)$. In view of the significant improvements achieved with the most recent data, updated numbers including preliminary results reported in the last $\tau$ Workshop [28] are also given.

\begin{tabular}{|c|c|c|}
\hline Parameter & PDG 94 & Montreux 94 \\
\hline$m_{\tau}$ & $\left(1777.1_{-0.5}^{+0.4}\right) \mathrm{MeV}$ & $(1777.0 \pm 0.3) \mathrm{MeV}$ \\
$\tau_{\tau}$ & $(295.6 \pm 3.1) \times 10^{-15} \mathrm{~s}$ & $(291.6 \pm 1.6) \times 10^{-15} \mathrm{~s}$ \\
$B_{e}$ & $(18.01 \pm 0.18) \%$ & $(17.79 \pm 0.09) \%$ \\
$B_{\mu}$ & $(17.65 \pm 0.24) \%$ & $(17.33 \pm 0.09) \%$ \\
$B_{\pi}$ & $(11.7 \pm 0.4) \%$ & $(11.09 \pm 0.15) \%$ \\
$B_{K}$ & $(0.67 \pm 0.23) \%$ & $(0.68 \pm 0.04) \%$ \\
$B_{h}$ & $(12.88 \pm 0.34) \%$ & $(11.77 \pm 0.14) \%$ \\
\hline
\end{tabular}

Table 4: World-average values [8] of the $\tau$ mass, lifetime, leptonic branching ratios and $\operatorname{Br}\left(\tau^{-} \rightarrow \nu_{\tau} \pi^{-} / K^{-}\right)$. The updated numbers in the third column, include preliminary results reported in the last $\tau$ Workshop [28].

\section{Universality tests}

The universality of the leptonic couplings can be tested through the ratios of the measured leptonic-decay widths:

$$
\frac{\Gamma_{\tau \rightarrow \mu}}{\Gamma_{\tau \rightarrow e}} \Longrightarrow\left|\frac{\widehat{G}_{\mu \tau}}{\widehat{G}_{e \tau}}\right|=\left\{\begin{array}{cc}
1.0038 \pm 0.0087 & (\text { PDG 94) } \\
1.0008 \pm 0.0036 & \text { (Montreux 94) }
\end{array},\right.
$$

2 The alternative is to go beyond the four-fermion Hamiltonian (11), allowing, for example, the decay of the tau into an electron and two (unobserved) neutral scalars, such as Majorons [27] or supersymmetric scalar neutrinos. 


$$
\frac{\Gamma_{\tau \rightarrow \mu}}{\Gamma_{\mu \rightarrow e}} \Longrightarrow\left|\frac{\widehat{G}_{\mu \tau}}{\widehat{G}_{e \mu}}\right|=\left\{\begin{array}{cc}
0.9970 \pm 0.0073 & (\text { PDG 94) } \\
0.9979 \pm 0.0037 & (\text { Montreux 94) }
\end{array}\right.
$$

where

$$
\widehat{G}_{l^{\prime} l} \equiv G_{l^{\prime} l} \sqrt{1+4 \eta_{l \rightarrow l^{\prime}} \frac{m_{l^{\prime}}}{m_{l}} \frac{g\left(m_{l^{\prime}}^{2} / m_{l}^{2}\right)}{f\left(m_{l^{\prime}}^{2} / m_{l}^{2}\right)}} .
$$

An important point, emphatically stressed by Fetscher and Gerber [29], concerns the extraction of $G_{e \mu}$ from $\mu$ decays, whose uncertainty is dominated by the uncertainty in $\eta$.

In models where $\eta=0, \widehat{G}_{l^{\prime} l}=G_{l^{\prime} l}$; then the limits (22) and (23) strongly constrain possible deviations from universality. To first-order in new physics, $G_{l^{\prime} l} \propto 1+\operatorname{Re}\left(\Delta g_{L L}^{V}\right)$. Therefore, at 90\% CL, $-0.005(-0.010)<\operatorname{Re}\left(\Delta g_{\mu_{L} \tau_{L}}^{V}-\Delta g_{e_{L} \tau_{L}}^{V}\right)<0.007(0.018)$ and $-0.008(-0.015)<\operatorname{Re}\left(\Delta g_{\mu_{L} \tau_{L}}^{V}-\Delta g_{e_{L} \mu_{L}}^{V}\right)<0.004$ (0.009), using the Montreux 94 (PDG 94) data.

Conversely, if lepton universality is assumed (i.e. $G_{l^{\prime} l}=G_{F}, g_{l_{\epsilon}^{\prime} l_{\omega}}^{n}=g_{\epsilon \omega}^{n}$ ), the leptonic decay ratios (22) and (23) provide limits on the low-energy parameter $\eta$. The best sensitivity [30] comes from $\widehat{G}_{\mu \tau}$, where the term proportional to $\eta$ is not suppressed by the small $m_{e} / m_{l}$ factor. The measured $B_{\mu} / B_{e}$ ratio implies then:

$$
\eta=\left\{\begin{array}{lc}
0.034 \pm 0.076 & (\text { PDG 94) } \\
0.007 \pm 0.033 & (\text { Montreux 94) }
\end{array} .\right.
$$

This determination is more accurate that the one in Table 2, obtained from the shape of the energy distribution, and is comparable to the value measured in $\mu$-decay: $\eta_{\mu \rightarrow e}=$ $-0.007 \pm 0.013$ [8].

A non-zero value of $\eta$ would show that there are at least two different couplings with opposite chiralities for the charged leptons. Since, we assume the V-A coupling $g_{L L}^{V}$ to be dominant, the second coupling would be [20] a Higgs type coupling $g_{R R}^{S}$ $\left[\eta \approx \operatorname{Re}\left(g_{R R}^{S}\right) / 2\right.$, to first-order in new-physics contributions]. Thus, Eq. (25) puts the (90\% CL) bound: $-0.09(-0.18)<\operatorname{Re}\left(g_{R R}^{S}\right)<0.12(0.32)$, using the Montreux 94 (PDG 94) data.

Finally, in models in which the new-physics couples exclusively to the lepton sector (so that the CKM matrix is unitary), further information may be found by comparing $G_{l^{\prime} l}$ with $G_{F}$ as extracted from the combination of $\beta$ and $K_{e 3}$ decays [31. Indeed, the usually quoted values for the CKM angles are extracted assuming that the coupling constant $g$, coupling $W$ to fermions, is the same for quarks and leptons. Thus, if there are new contributions affecting only the lepton couplings, any deviation from unitarity in the first row of the CKM matrix reflects a deviation of $g_{\mu}$ from the SM value.

\subsection{W-exchange model}

The universality constraints are commonly presented, assuming that the leptonic decays proceed exclusively through the SM V-A interaction. In that case the $\widehat{G}_{l^{\prime} l}$ ratios reduce 
to the corresponding ratios of leptonic $W$-couplings: $\left|\widehat{G}_{\mu \tau} / \widehat{G}_{e \tau}\right|=\left|g_{\mu} / g_{e}\right| ;\left|\widehat{G}_{\mu \tau} / \widehat{G}_{e \mu}\right|=$ $\left|g_{\tau} / g_{e}\right|$. Eq. (22) should then be compared with the more accurate value 32, 33.

$$
\left|\frac{g_{\mu}}{g_{e}}\right|=1.0017 \pm 0.0015
$$

obtained from the ratio $R_{e / \mu} \equiv \Gamma\left(\pi^{-} \rightarrow e^{-} \bar{\nu}_{e}\right) / \Gamma\left(\pi^{-} \rightarrow \mu^{-} \bar{\nu}_{\mu}\right)$.

The decay modes $\tau^{-} \rightarrow \nu_{\tau} \pi^{-}$and $\tau^{-} \rightarrow \nu_{\tau} K^{-}$can also be used to test universality through the ratios

$$
\begin{gathered}
R_{\tau / \pi} \equiv \frac{\Gamma\left(\tau^{-} \rightarrow \nu_{\tau} \pi^{-}\right)}{\Gamma\left(\pi^{-} \rightarrow \mu^{-} \bar{\nu}_{\mu}\right)}=\left|\frac{g_{\tau}}{g_{\mu}}\right|^{2} \frac{m_{\tau}^{3}}{2 m_{\pi} m_{\mu}^{2}} \frac{\left(1-m_{\pi}^{2} / m_{\tau}^{2}\right)^{2}}{\left(1-m_{\mu}^{2} / m_{\pi}^{2}\right)^{2}}\left(1+\delta R_{\tau / \pi}\right), \\
R_{\tau / K} \equiv \frac{\Gamma\left(\tau^{-} \rightarrow \nu_{\tau} K^{-}\right)}{\Gamma\left(K^{-} \rightarrow \mu^{-} \bar{\nu}_{\mu}\right)}=\left|\frac{g_{\tau}}{g_{\mu}}\right|^{2} \frac{m_{\tau}^{3}}{2 m_{K} m_{\mu}^{2}} \frac{\left(1-m_{K}^{2} / m_{\tau}^{2}\right)^{2}}{\left(1-m_{\mu}^{2} / m_{K}^{2}\right)^{2}}\left(1+\delta R_{\tau / K}\right),
\end{gathered}
$$

where the dependence on the hadronic matrix elements (the so-called decay constants $f_{\pi, K}$ ) factors out. Owing to the different energy scales involved, the radiative corrections to the $\tau^{-} \rightarrow \nu_{\tau} \pi^{-} / K^{-}$amplitudes are however not the same than the corresponding effects in $\pi^{-} / K^{-} \rightarrow \mu^{-} \bar{\nu}_{\mu}$. The size of the relative correction has been estimated by Marciano and Sirlin [34] to be $\delta R_{\tau / \pi}=(0.67 \pm 1) \$.$% , where the 1 \%$ error is due to the missing long-distance contributions to the tau decay rate. A recent evaluation of those long-distance corrections [35] quotes the more precise values

$$
\delta R_{\tau / \pi}=(0.16 \pm 0.14) \%, \quad \delta R_{\tau / K}=(0.90 \pm 0.22) \%
$$

Using these numbers, the measured $\tau^{-} \rightarrow \pi^{-} \nu_{\tau}$ and $\tau^{-} \rightarrow K^{-} \nu_{\tau}$ decay rates imply

$$
\left|\frac{g_{\tau}}{g_{\mu}}\right|_{\pi}=\left\{\begin{array}{l}
1.027 \pm 0.018 \\
1.006 \pm 0.008
\end{array} ; \quad\left|\frac{g_{\tau}}{g_{\mu}}\right|_{K}=\left\{\begin{array}{cc}
0.96 \pm 0.17 & (\text { PDG 94) } \\
0.972 \pm 0.029 & (\text { Montreux 94) }
\end{array} .\right.\right.
$$

The inclusive sum of both decay modes, i.e. $\Gamma\left[\tau^{-} \rightarrow h^{-} \nu_{\tau}\right]$ with $h=\pi, K$, provides a slightly more accurate determination:

$$
\left|\frac{g_{\tau}}{g_{\mu}}\right|_{\pi / K}=\left\{\begin{array}{cc}
1.043 \pm 0.015 & (\text { PDG 94) } \\
1.004 \pm 0.007 & (\text { Montreux 94) }
\end{array}\right.
$$

An independent test of lepton universality has been obtained at the $p-\bar{p}$ colliders, by comparing the ratios of the $\sigma \cdot B$ partial production cross-sections for the various $W^{-} \rightarrow l^{-} \bar{\nu}_{l}$ decay modes. The results of these analyses [36, 37, 38 are however less precise:

$$
\left|\frac{g_{\mu}}{g_{e}}\right|=1.00 \pm 0.08, \quad\left|\frac{g_{\tau}}{g_{e}}\right|=0.99 \pm 0.04
$$

Thus, the present data verify the universality of the leptonic charged-current couplings to the $0.16 \%(e / \mu)$ and $0.37 \%(\tau / \mu)$ level. The precision of the most recent $\tau$-decay measurements is becoming competitive with the more accurate $\pi$-decay determination. It is important to realize the complementarity of the different universality 
tests. The pure leptonic decay modes probe the charged-current couplings of a transverse $W$. In contrast, the decays $\pi / K \rightarrow l \bar{\nu}$ and $\tau \rightarrow \nu_{\tau} \pi / K$ are only sensitive to the longitudinal $W$ couplings. One can easily imagine new-physics scenarios which would modify differently the two types of leptonic couplings [39]. For instance, in the usual two-Higgs doublet model, the charged-scalar exchange generates a correction to the ratio $B_{\mu} / B_{e}$, but the pion-decay ratio $R_{e / \mu}$ remains unaffected. Similarly, lepton mixing between the $\nu_{\tau}$ and an hypothetical heavy neutrino would not modify the ratios $B_{\mu} / B_{e}$ and $R_{e / \mu}$, but would certainly correct the relation between $\Gamma\left(\tau^{-} \rightarrow \nu_{\tau} l^{-} \bar{\nu}_{l}\right)$ and $\Gamma\left(\mu^{-} \rightarrow \nu_{\mu} e^{-} \bar{\nu}_{e}\right)$.

\section{Constraints on new charged bosons}

In this section we assume that the interactions are mediated by charged vectors and/or charged scalars; therefore, there are no tensor couplings and Eqs. (7) become simpler. In particular, the quantities $\left(1-\frac{4}{3} \rho\right)$ and $\left(1-\frac{4}{3} \xi \delta\right)$ reduce to sums of $\left|g_{l_{\epsilon}^{\prime} l_{\omega}}^{n}\right|^{2}$, which are positive semidefinite; i.e., in the absence of tensor couplings, $\rho \leq \frac{3}{4}$ and $\xi \delta \leq \frac{3}{4}$. This allows us to extract direct bounds on several couplings.

The measured values of $\rho_{\mu \rightarrow e}, \rho_{\tau \rightarrow \mu}, \rho_{\tau \rightarrow e}$ and $\rho_{\tau \rightarrow l}(l=e, \mu)$ imply:

$$
\begin{aligned}
& \left|g_{e_{L} \mu_{R}}^{V}\right|^{2}+\left|g_{e_{R} \mu_{L}}^{V}\right|^{2}=-0.0024 \pm 0.0035<0.0045 \quad(90 \% \mathrm{CL}), \\
& \left|g_{\mu_{L} \tau_{R}}^{V}\right|^{2}+\left|g_{\mu_{R} \tau_{L}}^{V}\right|^{2}=0.016 \pm 0.051<0.094 \quad(90 \% \mathrm{CL}), \\
& \left|g_{e_{L} \tau_{R}}^{V}\right|^{2}+\left|g_{e_{R} \tau_{L}}^{V}\right|^{2}=0.019 \pm 0.037<0.074 \quad(90 \% \mathrm{CL}) \\
& \left|g_{l_{L} \tau_{R}}^{V}\right|^{2}+\left|g_{l_{R} \tau_{L}}^{V}\right|^{2}=0.023 \pm 0.029<0.064 \quad(90 \% \mathrm{CL}) \text {. }
\end{aligned}
$$

Except for $\left|g_{e_{L} \mu_{R}}^{V}\right|$, these limits are stronger than the general ones in Tables 1 and 3 .

Similarly, one gets from the different $\xi \delta$ measurements:

$$
\begin{aligned}
\left|g_{e_{L} \mu_{R}}^{V}\right|^{2}+\left|g_{e_{R} \mu_{L}}^{V}\right|^{2}+2\left|g_{e_{R} \mu_{R}}^{V}\right|^{2}+\frac{1}{2}\left|g_{e_{L} \mu_{R}}^{S}\right|^{2}+\frac{1}{2}\left|g_{e_{R} \mu_{R}}^{S}\right|^{2} & =-0.0017 \pm 0.0096 \\
\left|g_{\mu_{L} \tau_{R}}^{V}\right|^{2}+\left|g_{\mu_{R} \tau_{L}}^{V}\right|^{2}+2\left|g_{\mu_{R} \tau_{R}}^{V}\right|^{2}+\frac{1}{2}\left|g_{\mu_{L} \tau_{R}}^{S}\right|^{2}+\frac{1}{2}\left|g_{\mu_{R} \tau_{R}}^{S}\right|^{2} & =0.015 \quad(90 \% \mathrm{CL}) \\
\left|g_{e_{L} \tau_{R}}^{V}\right|^{2}+\left|g_{e_{R} \tau_{L}}^{V}\right|^{2}+2\left|g_{e_{R} \tau_{R}}^{V}\right|^{2}+\frac{1}{2}\left|g_{e_{L} \tau_{R}}^{S}\right|^{2}+\frac{1}{2}\left|g_{e_{R} \tau_{R}}^{S}\right|^{2} & =-0.36 \quad(90 \% \mathrm{CL}) \\
\left|g_{l_{L} \tau_{R}}^{V}\right|^{2}+\left|g_{l_{R} \tau_{L}}^{V}\right|^{2}+2\left|g_{l_{R} \tau_{R}}^{V}\right|^{2}+\frac{1}{2}\left|g_{l_{L} \tau_{R}}^{S}\right|^{2}+\frac{1}{2}\left|g_{l_{R} \tau_{R}}^{S}\right|^{2} & =-0.01 \pm 0.12 \\
& <0.19 \quad(90 \% \mathrm{CL}) .
\end{aligned}
$$

The limits on the $(\mu, e)$ couplings are weaker than the ones in Table 1. The bounds on the vector LR and RL couplings are also worse than the ones coming from Eq. (33). However, the resulting limits on the other couplings are stronger than the ones in Table 3. The constraint from $(\xi \delta)_{\tau \rightarrow e}$ shows explicitly that it is not possible to accommodate a value larger than $3 / 4$ with charged-boson (vector or/and scalar) exchanges.

In the absence of tensor couplings, we can combine the information on $\xi$ and $\rho$ to obtain another positive-semidefinite combination of couplings: $\left(1-\frac{4}{3} \rho\right)+\frac{1}{2}(1-\xi)$. The 
present data imply:

$$
\begin{aligned}
3\left|g_{e_{R} \mu_{L}}^{V}\right|^{2}+\left|g_{e_{R} \mu_{R}}^{V}\right|^{2}+\frac{1}{4}\left|g_{e_{L} \mu_{R}}^{S}\right|^{2}+\frac{1}{4}\left|g_{e_{R} \mu_{R}}^{S}\right|^{2} & =-0.0039 \pm 0.0053 \\
3\left|g_{\mu_{R} \tau_{L}}^{V}\right|^{2}+\left|g_{\mu_{R} \tau_{R}}^{V}\right|^{2}+\frac{1}{4}\left|g_{\mu_{L} \tau_{R}}^{S}\right|^{2}+\frac{1}{4}\left|g_{\mu_{R} \tau_{R}}^{S}\right|^{2} & =-0.0067 \quad(90 \% \mathrm{CL}), \\
3\left|g_{e_{R} \tau_{L}}^{V}\right|^{2}+\left|g_{e_{R} \tau_{R}}^{V}\right|^{2}+\frac{1}{4}\left|g_{e_{L} \tau_{R}}^{S}\right|^{2}+\frac{1}{4}\left|g_{e_{R} \tau_{R}}^{S}\right|^{2} & =0.16 \quad(90 \% \mathrm{CL}) \\
3\left|g_{l_{R} \tau_{L}}^{V}\right|^{2}+\left|g_{l_{R} \tau_{R}}^{V}\right|^{2}+\frac{1}{4}\left|g_{l_{L} \tau_{R}}^{S}\right|^{2}+\frac{1}{4}\left|g_{l_{R} \tau_{R}}^{S}\right|^{2} & =0.00 \pm 0.13 \\
& <0.10 \quad(90 \% \mathrm{CL}) \\
& <0.01 \pm 0.06
\end{aligned}
$$

The resulting limits on $\left|g_{e_{R} \mu_{L}}^{V}\right|,\left|g_{\mu_{R} \tau_{L}}^{V}\right|,\left|g_{\mu_{R} \tau_{R}}^{V}\right|,\left|g_{\mu_{L} \tau_{R}}^{S}\right|,\left|g_{\mu_{R} \tau_{R}}^{S}\right|,\left|g_{e_{R} \tau_{L}}^{V}\right|$ and $\left|g_{l_{R} \tau_{L}}^{V}\right|$ are stronger than the ones obtained before.

Combining the different limits, one gets the bounds shown in Table 5. The numbers with an asterisk have been derived from $(\xi \delta)_{e}$. If this information is not used, one finds the weaker limits: $\left|g_{e_{R} \tau_{R}}^{S}\right|<0.92,\left|g_{e_{L} \tau_{R}}^{S}\right|<0.92$ and $\left|g_{e_{R} \tau_{R}}^{V}\right|<0.46$.

\begin{tabular}{||c||c|l|l||l||}
\hline & $\mu \rightarrow e$ & $\tau \rightarrow \mu$ & $\tau \rightarrow e$ & $\tau \rightarrow l$ \\
\hline \hline$\left|g_{L L}^{S}\right|$ & $<0.55$ & $\leq 2$ & $\leq 2$ & $\leq 2$ \\
$\left|g_{R R}^{S}\right|$ & $<0.066$ & $<0.80$ & $<0.63^{*}$ & $<0.62$ \\
$\left|g_{L R}^{S}\right|$ & $<0.125$ & $<0.80$ & $<0.63^{*}$ & $<0.62$ \\
$\left|g_{R L}^{S}\right|$ & $<0.424$ & $\leq 2$ & $\leq 2$ & $\leq 2$ \\
\hline$\left|g_{L L}^{V}\right|$ & $>0.96$ & $\leq 1$ & $\leq 1$ & $\leq 1$ \\
$\left|g_{R R}^{V}\right|$ & $<0.033$ & $<0.40$ & $<0.32^{*}$ & $<0.31$ \\
$\left|g_{L R}^{V}\right|$ & $<0.060$ & $<0.31$ & $<0.27$ & $<0.25$ \\
$\left|g_{R L}^{V}\right|$ & $<0.047$ & $<0.23$ & $<0.27$ & $<0.18$ \\
\hline
\end{tabular}

Table 5: $90 \%$ CL limits for the couplings $g_{\epsilon \omega}^{n}$, assuming that there are no tensor couplings. The numbers with an asterisk use the measured value of $(\xi \delta)_{e}$.

Up to now, our only assumption has been the absence of tensor couplings. However, in many extensions of the SM, the bounds we have derived on the couplings can be improved due to additional knowledge of the underlying dynamics. Such is the case with any model whose deviations from the SM in the lepton sector are dominated by one intermediate state. This will typically occur with the least massive gauge boson, if its couplings are not suppressed by some approximate symmetry. In the following, we study the constraints associated with the addition of one 'dominant' intermediate boson to the SM.

\subsection{Factorization}

Let us assume that the interactions are mediated by a single charged boson (either vector or scalar). Then, the previous limits are improved due to additional relations 
among the couplings. Indeed, the factorization thus implied yields [9],

$$
\alpha_{L R}^{n} \alpha_{R L}^{n}=\alpha_{L L}^{n} \alpha_{R R}^{n}
$$

where we have used $\alpha$ (standing for $w, a, b$, etc.) to stress that these equations relate four-fermion effective couplings originating from the same boson intermediate state; $n=S$ for scalar mediated decays, and $n=V$ for vector mediated decays. These relations hold within any of the three channels, $(\mu, e),(\tau, e)$, and $(\tau, \mu)$.

Moreover, there are additional equations relating different processes, such as

$$
\begin{aligned}
& \alpha_{\mu_{L} \tau_{L}}^{n} \alpha_{e_{L} \tau_{R}}^{n}=\alpha_{\mu_{L} \tau_{R}}^{n} \alpha_{e_{L} \tau_{L}}^{n}, \\
& \alpha_{\mu_{L} \tau_{L}}^{n} \alpha_{e_{L} \mu_{R}}^{n *}=\alpha_{\mu_{R} \tau_{L}}^{n} \alpha_{e_{L} \mu_{L}}^{n *}, \\
& \alpha_{e_{L} \tau_{L}}^{n} \alpha_{e_{R} \mu_{L}}^{n}=\alpha_{e_{R} \tau_{L}}^{n} \alpha_{e_{L} \mu_{L}},
\end{aligned}
$$

and

$$
\operatorname{Im}\left(\alpha_{e_{\epsilon} \mu_{\lambda}}^{n} \alpha_{e_{\epsilon} \tau_{\gamma}}^{n *} \alpha_{\mu_{\lambda} \tau_{\gamma}}^{n}\right)=0
$$

for any chosen set of chiralities $(\epsilon, \lambda, \gamma)$. Other similar equations may be obtained from these with the help of Eq. (36). Most of these relations constrain pairs of variables to the space below a hyperbola.

\section{$5.2 \quad$ Non-standard $W$ interactions}

In this case we consider only $W$-mediated interactions but admitting the possibility that the $W$ couples non-universally to leptons of any chirality. Then,

$$
g_{\epsilon \omega}^{V} \equiv w_{\epsilon \omega}^{V} \text {. }
$$

while all other couplings vanish, leading to $\eta=0$. The normalization condition $N=1$, implies strong $(90 \% \mathrm{CL})$ lower bounds on the $g_{L L}^{V}$ couplings:

$$
\left|g_{e_{L} \mu_{L}}^{V}\right|>0.997 ;\left|g_{\mu_{L} \tau_{L}}^{V}\right|>0.83 ;\left|g_{e_{L} \tau_{L}}^{V}\right|>0.87^{*}(0.80) ;\left|g_{l_{L} \tau_{L}}^{V}\right|>0.90 \text {. }
$$

The two $\left|g_{e_{L} \tau_{L}}^{V}\right|$ limits correspond to the results obtained using the $(\xi \delta)_{e}$ measurement $(*)$, or ignoring it (number within brackets).

Since in this case the lower bounds of Eq. (40) are direct limits on the couplings, $w_{L L}^{V}$, of the intermediate boson under study, we can use the factorization equation (36), rewritten in the form,

$$
\left|w_{e_{R} \mu_{R}}^{V}\right|=\left|\frac{w_{e_{L} \mu_{R}}^{V} w_{e_{R} \mu_{L}}^{V}}{w_{e_{L} \mu_{L}}^{V}}\right|<0.0028,
$$

to improve the bound on $g_{e_{R} \mu_{R}}^{V}=w_{e_{R} \mu_{R}}^{V}$ by an order of magnitude.

For the $(\tau, \mu)$ channel, we can use the lower bound on $g_{\mu_{L} \tau_{L}}^{V}$, together with the factorization relations among the couplings of different channels to get the improved (90\% CL) limits:

$$
\left|w_{\mu_{R} \tau_{L}}^{V}\right|=\left|\frac{w_{\mu_{L} \tau_{L}}^{V} w_{e_{L} \mu_{R}}^{V *}}{w_{e_{L} \mu_{L}}^{V *}}\right|<0.060 ; \quad\left|w_{\mu_{R} \tau_{R}}^{V}\right|=\left|\frac{w_{\mu_{L} \tau_{R}}^{V} w_{e_{L} \mu_{R}}^{V *}}{w_{e_{L} \mu_{L}}^{V *}}\right|<0.019 .
$$


Similarly, for the $(\tau, e)$ channel we find

$$
\left|w_{e_{R} \tau_{L}}^{V}\right|=\left|\frac{w_{e_{L} \tau_{L}}^{V} w_{e_{R} \mu_{L}}^{V}}{w_{e_{L} \mu_{L}}^{V}}\right|<0.047 ; \quad\left|w_{e_{R} \tau_{R}}^{V}\right|=\left|\frac{w_{e_{L} \tau_{R}}^{V} w_{e_{R} \mu_{L}}^{V}}{w_{e_{L} \mu_{L}}^{V}}\right|<0.013 .
$$

Notice that no information on $(\xi \delta)_{e}$ has been used here. Thus, for the case of nonstandard $W$-mediated interactions, the relations among channels developed above allow us to improve the limits on some couplings by one order of magnitude.

Using the bounds (42) and (43), the normalization condition $N=1$ allows us to further improve the $(90 \% \mathrm{CL})$ lower limits on the $w_{l_{L} \tau_{L}}^{V}$ couplings

$$
\left|w_{\mu_{L} \tau_{L}}^{V}\right|>0.95 ; \quad\left|w_{e_{L} \tau_{L}}^{V}\right|>0.96
$$

where the last bound is now independent of the $(\xi \delta)_{e}$ measurement.

Table 6 summarizes the limits on $W$-mediated interactions.

\begin{tabular}{||l||l|l|l||}
\hline & \multicolumn{1}{|c||}{$\mu \rightarrow e$} & \multicolumn{1}{|c||}{$\rightarrow \mu$} & $\tau \rightarrow e$ \\
\hline \hline$\left|w_{L L}^{V}\right|$ & $>0.997$ & $>0.95$ & $>0.96$ \\
$\left|w_{R R}^{V}\right|$ & $<0.0028$ & $<0.019$ & $<0.013$ \\
$\left|w_{L R}^{V}\right|$ & $<0.060$ & $<0.31$ & $<0.27$ \\
$\left|w_{R L}^{V}\right|$ & $<0.047$ & $<0.060$ & $<0.047$ \\
\hline
\end{tabular}

Table 6: $90 \%$ CL limits for the $w_{\epsilon \omega}^{V}$ couplings, assuming that any additional interactions are negligible.

\subsection{SM plus Charged Vector}

If in addition to the SM $W$ boson $\left(w_{L L}^{V} \neq 0\right.$, and all others zero), there is another vector boson with a mass not too large, then its presence will be constrained by the effective vector couplings $\left(a_{\epsilon \omega}^{V}\right)$ that it generates. In particular, we have seen in Sect. \& that differences of

$$
g_{L L}^{V}=w_{L L}^{V}+a_{L L}^{V}
$$

corresponding to different channels are well constrained by universality tests.

The general analysis follows the one of the previous case, except for the fact that Eqs. (41), (42) and (43) do not provide upper bounds on the single couplings on the left hand side. Indeed, the lower bound on $g_{e_{L} \mu_{L}}^{V}$, which affects the sum of the SM with the new contribution, does not translate into a lower bound for $a_{e_{L} \mu_{L}}^{V}$. This is just a reflection of the fact that the experiments are consistent with the inexistence of a contribution from a new vector boson. Of course, those relations are still useful in the form of Eqs. (37), to limit products of couplings. What we cannot do in this case, is use these relations, together with the lower bound on $g_{e_{L} \mu_{L}}^{V}$, to place limits on a single coupling. 
For instance,

$$
\begin{aligned}
& \left|a_{e_{L} \mu_{L}}^{V} g_{e_{R} \mu_{R}}^{V}\right|=\left|g_{e_{L} \mu_{R}}^{V} g_{e_{R} \mu_{L}}^{V}\right|<0.0028 \quad(90 \% \mathrm{CL}), \\
& \left|a_{\mu_{L} \tau_{L}}^{V} g_{\mu_{R} \tau_{R}}^{V}\right|=\left|g_{\mu_{L} \tau_{R}}^{V} g_{\mu_{R} \tau_{L}}^{V}\right|<0.071 \quad(90 \% \mathrm{CL}), \\
& \left|a_{e_{L} \tau_{L}}^{V} g_{e_{R} \tau_{R}}^{V}\right|=\left|g_{e_{L} \tau_{R}}^{V} g_{e_{R} \tau_{L}}^{V}\right|<0.073 \quad(90 \% \mathrm{CL}) \text {. }
\end{aligned}
$$

These equations establish non-trivial constraints since they involve $a_{L L}^{V}$, to which we do not have direct experimental access. So, in addition to direct bounds on individual magnitudes, we have also constrained the allowed values to the space below a hyperbola, in the respective plane. Of course, there are many such constraints. Here we just want to illustrate their existence and point out that these constraints translate into nontrivial information and might be especially useful in specific models that have a small number of parameters.

\subsection{SM plus Charged Scalar}

In this case $\rho=3 / 4$ and

$$
\begin{aligned}
2 \eta & =\operatorname{Re}\left(w_{L L}^{V} g_{R R}^{S *}\right) \sim \operatorname{Re}\left(g_{R R}^{S}\right) \\
2(1-\xi) & =2\left(1-\frac{4}{3} \xi \delta\right)=\left|g_{L R}^{S}\right|^{2}+\left|g_{R R}^{S}\right|^{2}
\end{aligned}
$$

The positivity of $(1-\xi)$ leads now to slightly improved (90\% CL) bounds for the scalar couplings,

$$
\begin{gathered}
\left|g_{e_{L} \mu_{R}}^{S}\right|^{2}+\left|g_{e_{R} \mu_{R}}^{S}\right|^{2}=-0.006 \pm 0.016<0.023, \\
\left|g_{\mu_{L} \tau_{R}}^{S}\right|^{2}+\left|g_{\mu_{R} \tau_{R}}^{S}\right|^{2}=-0.46 \pm 0.48<0.56 \\
\left|g_{e_{L} \tau_{R}}^{S}\right|^{2}+\left|g_{e_{R} \tau_{R}}^{S}\right|^{2}=-0.06 \pm 0.50<0.79 \\
\left|g_{l_{L} \tau_{R}}^{S}\right|^{2}+\left|g_{l_{R} \tau_{R}}^{S}\right|^{2}=-0.12 \pm 0.22<0.30 .
\end{gathered}
$$

The limits on the $(\mu, e)$ couplings are still weaker than the ones in Table 1, but the others are stronger than the ones in Eqs. (34) and (35). Only the bound obtained from $(\xi \delta)_{e}$ is better.

The information on the low-energy parameter $\eta$ gives the $(90 \% \mathrm{CL})$ limits:

$$
\begin{aligned}
-0.057 & <\operatorname{Re}\left(w_{e_{L} \mu_{L}}^{V} g_{e_{R} \mu_{R}}^{S *}\right)<0.029 \\
-1.03 & <\operatorname{Re}\left(w_{\mu_{L} \tau_{L}}^{V} g_{\mu_{R} \tau_{R}}^{S *}\right)<0.47 .
\end{aligned}
$$

Assuming lepton universality, Eq. (25) yields a much better bound on the $\tau \rightarrow l$ couplings:

$$
\left(\begin{array}{c}
-0.18 \\
-0.09
\end{array}\right)<\operatorname{Re}\left(w_{l_{L} \tau_{L}}^{V} g_{l_{R} \tau_{R}}^{S *}\right)<\left(\begin{array}{c}
0.32 \\
0.12
\end{array}\right) \quad\left(\begin{array}{c}
\text { PDG 94 } \\
\text { Montreux 94 }
\end{array}\right)
$$

which, however, is still worse than the limit obtained from $\eta_{\mu \rightarrow e}$. 
Using the factorization relations, one gets additional limits, such as

$$
\begin{aligned}
& \left|g_{e_{L} \mu_{R}}^{S} g_{e_{R} \mu_{L}}^{S}\right|=\left|g_{e_{L} \mu_{L}}^{S} g_{e_{R} \mu_{R}}^{S}\right|<3.6 \times 10^{-2}, \\
& \left|g_{e_{R} \mu_{L}}^{S} g_{\mu_{R} \tau_{R}}^{S}\right|=\left|g_{e_{R} \mu_{R}}^{S} g_{\mu_{L} \tau_{R}}^{S}\right|<0.050 .
\end{aligned}
$$

improving the limits on the products in the left-hand side of the equations over the bounds obtainable directly from Table 5. At present, the $\tau_{L}$ couplings are only constrained by the normalization condition: $\left|g_{l_{\epsilon} \tau_{L}}^{S}\right|<2$ and $\left|g_{l_{L} \tau_{L}}^{V}\right|<1$.

\section{Constraints on new neutral bosons}

In this section we study the possible existence of neutral bosons violating the leptonic $l$ and $l^{\prime}$ numbers. For example, in models with heavier leptons with non-canonical quantum number assignments, there are non-diagonal $Z^{0}$ interactions induced by the mixing of the standard leptons with exotic ones. In other models, similar couplings with new neutral scalars arise naturally at levels close to the current experimental values [31.

Of course, such interactions will also contribute to the well constrained flavourviolating decays into three charged leptons, such as $\mu \rightarrow e e e$. The $l^{-} \rightarrow \nu_{l} l^{\prime-} \bar{\nu}_{l^{\prime}}$ decays involve two charged-lepton and two neutrino couplings to the intermediate boson, while decays of the type $l^{-} \rightarrow l_{1}^{-} l_{2}^{+} l_{3}^{-}$involve four charged-lepton couplings. Therefore, the two types of decay provide complementary information. Note, however, that in many models the neutrino and charged-lepton couplings are related; in such cases, the constraints from the $l^{-} \rightarrow l_{1}^{-} l_{2}^{+} l_{3}^{-}$decays are usually much stronger than those obtained from the $l^{-} \rightarrow \nu_{l} l^{\prime-} \bar{\nu}_{l^{\prime}}$ spectra.

It is easily shown that if, as we are assuming, the final neutrinos are massless and not observed, one falls back on an effective hamiltonian like that of Eq. (1), even in the presence of lepton-number nonconservation [26]. In the appendix, this is shown explicitly for the case of neutral boson mediated interactions. We also include there the derivation of some formulae useful in this section, and a discussion of bounds from neutrinoless charged-lepton decays. In the cases studied here there are no relations among different channels.

\subsection{SM plus Neutral Vector}

When the decay is mediated by neutral vector bosons, all the LR and RL couplings vanish and $\rho=3 / 4$. Since there are no tensor couplings, the relevant bounds on Table 5 are also valid in this case. Moreover, $(1-\xi)$ is now a positive-semidefinite quantity, which gives the additional (90\% CL) limits,

$$
\begin{aligned}
& \frac{1}{2}\left|g_{e_{R} \mu_{R}}^{S}\right|^{2}+2\left|g_{e_{R} \mu_{R}}^{V}\right|^{2}<0.011 \\
& \frac{1}{2}\left|g_{\mu_{R} \tau_{R}}^{S}\right|^{2}+2\left|g_{\mu_{R} \tau_{R}}^{V}\right|^{2}<0.28 \\
& \frac{1}{2}\left|g_{e_{R} \tau_{R}}^{S}\right|^{2}+2\left|g_{e_{R} \tau_{R}}^{V}\right|^{2}<0.39 \\
& \frac{1}{2}\left|g_{l_{R} \tau_{R}}^{S}\right|^{2}+2\left|g_{l_{R} \tau_{R}}^{V}\right|^{2}<0.15
\end{aligned}
$$


The limits on the $(\mu, e)$ couplings are weaker than the ones in Table 1, but the others are stronger than the ones in Eqs. (34) and (35). Only the bound obtained from $(\xi \delta)_{e}$ is better.

As usual, we distinguish the SM $W$ and the neutral vector boson contributions to $g_{L L}^{V}$ by the letters $w$ and $a$, respectively. Hence,

$$
g_{L L}^{V}=w_{L L}^{V}+a_{L L}^{V}
$$

As shown in the appendix A.1, the new contributions satisfy the relation

$$
a_{L L}^{S} a_{R R}^{S}=4 a_{L L}^{V} a_{R R}^{V}
$$

which yields the $90 \%$ CL bounds

$$
\begin{aligned}
\left|a_{e_{L} \mu_{L}}^{V} g_{e_{R} \mu_{R}}^{V}\right| & =\frac{1}{4}\left|g_{e_{L} \mu_{L}}^{S} g_{e_{R} \mu_{R}}^{S}\right|<9.1 \times 10^{-3} \\
\left|a_{\mu_{L} \tau_{L}}^{V} g_{\mu_{R} \tau_{R}}^{V}\right| & =\frac{1}{4}\left|g_{\mu_{L} \tau_{L}}^{S} g_{\mu_{R} \tau_{R}}^{S}\right|<0.37 \\
\left|a_{e_{L} \tau_{L}}^{V} g_{e_{R} \tau_{R}}^{V}\right| & =\frac{1}{4}\left|g_{e_{L} \tau_{L}}^{S} g_{e_{R} \tau_{R}}^{S}\right|<0.32^{*}(0.44)
\end{aligned}
$$

\begin{tabular}{|c|c|c|c|}
\hline & $\mu \rightarrow e$ & $\tau \rightarrow \mu$ & $\tau \rightarrow e$ \\
\hline$\left|\alpha_{l^{\prime} l}\right|^{2} \sum_{m, n}\left|\theta_{m n}\right|^{2}$ & $<1.1 \times 10^{-3}$ & $<0.14$ & $<0.20$ \\
\hline$\left|\beta_{l^{\prime} l}\right|^{2} \quad \sum_{m, n}\left|\theta_{m n}\right|^{2}$ & $<7.6 \times 10^{-2}$ & - & - \\
\hline
\end{tabular}

Again, these relations yield constraints on $a_{L L}^{V}$, to which there is no direct experimental access.

Table 7: 90\% CL limits on products of quadratic polynomials in the lepton and neutrino couplings. If one uses the measured value of $(\xi \delta)_{e}$, the number on the last column will read instead 0.10 .

Assuming that the neutrinos are not detected, the neutral-vector-induced effective couplings may be written as

$$
\begin{aligned}
& a_{l_{R}^{\prime} l_{R}}^{V}=\alpha_{l^{\prime} l}\left[\sum_{m, n}\left|\theta_{m n}\right|^{2}\right]^{1 / 2}, \quad a_{l_{R}^{\prime} l_{R}}^{S}=-2 \alpha_{l^{\prime} l}\left[\sum_{m, n}\left|\sigma_{m n}\right|^{2}\right]^{1 / 2}, \\
& a_{l_{L}^{\prime} l_{L}}^{V}=\beta_{l^{\prime} l}\left[\sum_{m, n}\left|\sigma_{m n}\right|^{2}\right]^{1 / 2}, \\
& a_{l_{L}^{\prime} l_{L}}^{S}=-2 \beta_{l^{\prime} l}\left[\sum_{m, n}\left|\theta_{m n}\right|^{2}\right]^{1 / 2},
\end{aligned}
$$

where $\alpha(\beta)$ is the hermitian coupling matrix of the right- (left-) handed charged leptons to the neutral vector and $\theta(\sigma)$ is the coupling matrix of the right- (left-) handed neutrinos, in appropriate units (see appendix A.1). The experimental limits on the effective four-fermion couplings constrain then these combinations of the original vector couplings. We summarize these results in Table 0 . The bounds on the first line remain the same with $\theta$ substituted by $\sigma$ and the missing numbers on the second line are due to the lack of experimental access to $Q_{\tau_{L}}$. 


\subsection{SM plus Neutral Scalars}

Finally, we consider the case in which there is a neutral scalar contribution to $\mu$ and $\tau$ leptonic decays, in addition to the SM contribution. These new contributions vanish for the LL and RR couplings and satisfy the relations

$$
a_{L R}^{V}=a_{L R}^{S}=2 a_{L R}^{T} \quad ; \quad a_{R L}^{V}=a_{R L}^{S}=2 a_{R L}^{T} .
$$

This allows us to express everything in terms of the vector couplings. One gets then the positive definite quantities:

$$
1-\frac{4}{3} \rho=1-\frac{4}{3} \xi \delta=2\left(\left|g_{L R}^{V}\right|^{2}+\left|g_{R L}^{V}\right|^{2}\right)
$$

and

$$
\left(1-\frac{4}{3} \rho\right)+\frac{1}{2}(1-\xi)=6\left|g_{R L}^{V}\right|^{2}
$$

The $N=1$ constraint provides the additional relation

$$
1=\left|g_{L L}^{V}\right|^{2}+2\left(\left|g_{L R}^{V}\right|^{2}+\left|g_{R L}^{V}\right|^{2}\right) .
$$

Thus, $1-\frac{4}{3} \rho=1-\frac{4}{3} \xi \delta=\left(1-\left|g_{L L}^{V}\right|^{2}\right)$, which gives lower bounds on all $g_{L L}^{V}$ couplings. The resulting $90 \%$ CL limits are given in Table 8 .

\begin{tabular}{||c||c|c|c||c||}
\hline & $\mu \rightarrow e$ & $\tau \rightarrow \mu$ & $\tau \rightarrow e$ & $\tau \rightarrow l$ \\
\hline \hline$\left|g_{L L}^{V}\right|$ & $>0.998$ & $>0.95$ & $>0.96$ & $>0.97$ \\
$\left|g_{L R}^{V}\right|$ & $<0.047$ & $<0.22$ & $<0.19$ & $<0.18$ \\
$\left|g_{R L}^{V}\right|$ & $<0.033$ & $<0.16$ & $<0.19$ & $<0.13$ \\
\hline
\end{tabular}

Table 8: $90 \%$ CL limits for the $g_{\epsilon \omega}^{n}$ couplings, taking $g_{R R}^{n}=0, g_{L L}^{S}=0, g_{L R}^{V}=g_{L R}^{S}=$ $2 g_{L R}^{T}$ and $g_{R L}^{V}=g_{R L}^{S}=2 g_{R L}^{T}$.

In addition we have the constraints from $\eta$, which at $90 \%$ CL give

$$
\begin{aligned}
-0.007 & <\operatorname{Re}\left(g_{e_{L} \mu_{R}}^{V} g_{e_{R} \mu_{L}}^{V *}\right)<0.004 \\
-0.13 & <\operatorname{Re}\left(g_{\mu_{L} \tau_{R}}^{V} g_{\mu_{R} \tau_{L}}^{V *}\right)<0.06 .
\end{aligned}
$$

These effective couplings may be written in terms of the ones in the original lagrangian as

$$
a_{l_{R}^{\prime} l_{L}}^{V}=A_{l^{\prime} l}\left[\sum_{m, n}\left|B_{m n}\right|^{2}\right]^{1 / 2}, \quad a_{l_{L}^{\prime} l_{R}}^{V}=A_{l l^{\prime}}^{*}\left[\sum_{m, n}\left|B_{m n}\right|^{2}\right]^{1 / 2},
$$

where $A(B)$ is the coupling matrix of the charged leptons (neutrinos) to the neutral scalar, in appropriate units (see appendix A.2). So, the previous limits contain combined information from the two sectors.

It is important to emphasize that, within the philosophy we sustain of discarding intermediate tensor particles (for they hardly appear in any reasonable model beyond the SM), this is the only possible source of tensorial terms. This fact has interesting consequences which we will explore in the next section. 


\begin{tabular}{|c|c|c|c|c|}
\hline & $\begin{array}{c}\text { SM + Charged } \\
\text { Vector } \\
\text { Nonstandard } W\end{array}$ & $\begin{array}{c}\text { SM + Charged } \\
\text { Scalar }\end{array}$ & $\begin{array}{c}\text { SM + Neutral } \\
\text { Vector }\end{array}$ & $\begin{array}{c}\text { SM + Neutral } \\
\text { Scalar }\end{array}$ \\
\hline$\rho-3 / 4$ & $<0$ & $\mathrm{SM}$ & $\mathrm{SM}$ & $<0$ \\
\hline$\xi-1$ & $\mathrm{AS}$ & $<0$ & $<0$ & $\mathrm{AS}$ \\
\hline$(\delta \xi)-3 / 4$ & $<0$ & $<0$ & $<0$ & $<0$ \\
\hline$\eta$ & $\mathrm{SM}$ & $\mathrm{AS}$ & $\mathrm{AS}$ & $\mathrm{AS}$ \\
\hline
\end{tabular}

Table 9: Theoretical constraints on the Michel parameters

\section{Opportunities for Physics Beyond the SM}

In Table 9 we present a summary of the theoretical constraints imposed on the measured quantities, for the various cases under study. There, SM denotes that the Standard Model results are recovered and AS indicates that any sign is allowed.

It is immediately apparent that $\rho \leq 3 / 4$ and $(\delta \xi)<3 / 4$ in all cases that we have studied. Thus one can have new physics and still $\rho$ be equal to the SM value. In fact, any interaction consisting of an arbitrary combination of $g_{\epsilon \omega}^{S}$ 's and $g_{\gamma \gamma}^{V}$ 's yields this result [20]. On the other hand, $(\delta \xi)$ will be different from $3 / 4$ in any of the cases above providing, in principle, a better opportunity for the detection of Physics Beyond the SM.

The above features are easy to understand by looking back at Eqs. (7) and recalling that the tensor couplings can only be generated by neutral scalar interactions (violating individual lepton flavours), in which case they are proportional to the scalar couplings. It is easy to see that having two such neutral scalars will not alter the situation. Indeed, to obtain $\rho>3 / 4$ or $(\delta \xi)>3 / 4$ one will also need the presence of a charged scalar.

Let's then assume that we have a neutral scalar with couplings

$$
a_{L R}^{V}=a_{L R}^{S}=2 a_{L R}^{T} \quad ; \quad a_{R L}^{V}=a_{R L}^{S}=2 a_{R L}^{T},
$$

and a charged scalar with couplings $b_{\epsilon \omega}^{S}$. We obtain,

$$
\begin{aligned}
\rho-\frac{3}{4}= & -\frac{3}{4}\left[2\left|a_{L R}^{S}\right|^{2}+2\left|a_{R L}^{S}\right|^{2}+\frac{1}{2} \operatorname{Re}\left(a_{L R}^{S} b_{L R}^{S *}+a_{R L}^{S} b_{R L}^{S *}\right)\right], \\
(\xi \delta)-\frac{3}{4}=- & \frac{3}{4}\left[\frac{1}{2}\left|b_{R R}^{S}\right|^{2}+\frac{1}{8}\left|a_{L R}^{S}-b_{L R}^{S}\right|^{2}+\frac{3}{8}\left|a_{L R}^{S}+b_{L R}^{S}\right|^{2}\right. \\
& \left.+\frac{3}{2}\left|a_{L R}^{S}\right|^{2}+2\left|a_{R L}^{S}\right|^{2}+\frac{1}{2} \operatorname{Re}\left(a_{R L}^{S} b_{R L}^{S *}\right)\right] .
\end{aligned}
$$

The first equation shows that $\rho$ might exceed $3 / 4$ provided that

$$
\operatorname{Re}\left(\frac{b_{L R}^{S}}{a_{L R}^{S}}\right)<-4,
$$

or

$$
\operatorname{Re}\left(\frac{b_{R L}^{S}}{a_{R L}^{S}}\right)<-4
$$


As for $(\delta \xi)$, it can only exceed the SM value through $R L$ couplings, and only if the last equation is satisfied. Then, detecting $\rho$ greater than the SM value would mean that there were at least a charged scalar and a neutral scalar in action. A measurement of $(\delta \xi)$ greater than $3 / 4$ would then discriminate between $R L$ and $L R$ couplings. However, as pointed out before, a measurement of $(\delta \xi)>3 / 4$ must, in general, be accompanied by a measurement of $\xi>1$. If the contrary were to become well established, we would have detected physics beyond the four-fermion hamiltonian.

\section{Conclusions}

We have used the recent measurements on the Michel parameters in tau decays to perform a complete, model independent analysis of the constraints implied for scalar and neutral bosons, as they exist in most models beyond the SM. If the new contributions are dominated by the effect of one such new intermediate boson, relations among the different couplings arise.

In the case of charged intermediate bosons, these relations involve couplings from different decays. If the most important new feature is the coupling of the usual $W$ boson with right handed leptons, then the data from muon neutrino scattering off electrons can be used to improve some of the limits on couplings in tau decays by an order of magnitude. In the other cases, it constrains products of couplings of different channels to the space below a hyperbola. This information will be particularly useful for models in which these couplings are functions of the same parameters of the original theory.

In case the dominant new features are provided by the exchange of flavour violating neutral scalars, there are no relations among the different channels. The relations within each channel were derived assuming that the final neutrinos are massless and not observed. This shows explicitly that the analysis based on the common four fermion hamiltonian is still valid in this case. It is shown in the appendix that, given the current experimental situation, the bounds obtained from the Michel parameters only compete with those provided by the decays $l^{-} \rightarrow l_{1}^{-} l_{2}^{+} l_{3}^{-}$, in theories were the charged lepton couplings to the intermediate particle carrying flavour are suppressed by some (exact or approximate) symmetry.

\section{Acknowledgements}

This work has been supported in part by CICYT (Spain) under grant No. AEN93-0234. The work of J.P.S. was funded by the E.U. under the Human Capital and Mobility Program. He is indebted to G.C. Branco, L. Lavoura and Instituto Superior Técnico for their kind hospitality, and to J. Raab and A. Stahl for useful discussions.

\section{A Flavour-violating neutral-mediated interactions}




\section{A.1 Neutral Vector Bosons}

We parametrize the interaction of a neutral vector boson $V_{\mu}^{0}$ with the leptons by

$$
\mathcal{L}=J^{\mu} V_{\mu}^{0}
$$

where

$$
J^{\mu}=\left(M_{V} \sqrt{2 \sqrt{2} G_{F}}\right)\left[\bar{l} \gamma^{\mu}\left(\alpha \gamma_{R}+\beta \gamma_{L}\right) l+\bar{\nu} \gamma^{\mu}\left(\theta \gamma_{R}+\sigma \gamma_{L}\right) \nu\right] .
$$

$M_{V}$ is the mass of the neutral vector, $\gamma_{R, L} \equiv\left(1 \pm \gamma_{5}\right) / 2$ are the chirality projectors, and $\alpha, \beta, \theta$ and $\sigma$ are $3 \times 3$ hermitian matrices in the respective flavour spaces.

Since $M_{V}$ is typically much larger than the energy scale of $\mu$ and $\tau$ decays, the interaction is effectively that of four fermions,

$$
\mathcal{H}=\frac{1}{2 M_{V}^{2}} J^{\mu} J_{\mu}^{\dagger}
$$

which, after Fierzing, yields an effective interaction between two neutrinos and two charged leptons of the form,

$$
\begin{aligned}
\mathcal{H}=\frac{4 G_{F}}{\sqrt{2}} & {\left[\alpha_{l^{\prime} l} \theta_{m n}\left(\overline{l_{R}^{\prime}} \gamma^{\mu} \nu_{R}^{n}\right)\left(\overline{\nu_{R}^{m}} \gamma_{\mu} l_{R}\right)+\beta_{l^{\prime} l} \sigma_{m n}\left(\overline{l_{L}^{\prime}} \gamma^{\mu} \nu_{L}^{n}\right)\left(\overline{\nu_{L}^{m}} \gamma_{\mu} l_{L}\right)\right.} \\
& \left.-2 \alpha_{l^{\prime} l} \sigma_{m n}\left(\overline{l_{R}^{\prime}} \nu_{L}^{n}\right)\left(\overline{\nu_{L}^{m}} l_{R}\right)-2 \beta_{l^{\prime} l} \theta_{m n}\left(\overline{l_{L}^{\prime}} \nu_{R}^{n}\right)\left(\overline{\nu_{R}^{m}} l_{L}\right)\right] .
\end{aligned}
$$

As expected, the chirality changing couplings RL and LR vanish. Notice that the resulting effective couplings satisfy the relation

$$
a_{l_{R}^{\prime} l_{R}}^{S} a_{l_{L}^{\prime} l_{L}}^{S}=4 a_{l_{R}^{\prime} l_{R}}^{V} a_{l_{L}^{\prime} l_{L}}^{V}
$$

Since the neutrinos are unobserved, Eq. (70) reduces to the hamiltonian (11), with effective couplings:

$$
\begin{array}{ll}
a_{l_{R}^{\prime} l_{R}}^{V}=\alpha_{l^{\prime} l} \Theta \quad, & a_{l_{R}^{\prime} l_{R}}^{S}=-2 \alpha_{l^{\prime} l} \Sigma, \\
a_{l_{L}^{\prime} l_{L}}^{V}=\beta_{l^{\prime} l} \Sigma & , \quad a_{l_{L}^{\prime} l_{L}}^{S}=-2 \beta_{l^{\prime} l} \Theta,
\end{array}
$$

where

$$
\Theta=\left[\sum_{m, n}\left|\theta_{m n}\right|^{2}\right]^{1 / 2}, \quad \Sigma=\left[\sum_{m, n}\left|\sigma_{m n}\right|^{2}\right]^{1 / 2} .
$$

The lagrangian (67) also induces flavour-changing decays of $\mu$ and $\tau$ into three charged leptons. Neglecting the masses of the final leptons, the corresponding decay widths can be written as

$$
\Gamma\left[l^{-} \rightarrow l_{1}^{-} l_{2}^{+} l_{3}^{-}\right]=\frac{m_{l}^{5} G_{F}^{2}}{192 \pi^{3}} \mathcal{C}_{l_{1} l_{2} l_{3}}^{l},
$$

where $\mathcal{C}_{l_{1} l_{2} l_{3}}^{l}$ are quartic polynomials in the lepton couplings $\alpha$ and $\beta$, given by

$$
\begin{aligned}
\mathcal{C}_{l_{1} l_{2} l_{3}}^{l}=w_{s} & \left\{\left(\left|\alpha_{l_{1} l}\right|^{2}+\left|\beta_{l_{1} l}\right|^{2}\right)\left(\left|\alpha_{l_{3} l_{2}}\right|^{2}+\left|\beta_{l_{3} l_{2}}\right|^{2}\right)\right. \\
& +\left(\left|\alpha_{l_{3} l}\right|^{2}+\left|\beta_{l_{3} l}\right|^{2}\right)\left(\left|\alpha_{l_{1} l_{2}}\right|^{2}+\left|\beta_{l_{1} l_{2}}\right|^{2}\right) \\
& \left.+2 \operatorname{Re}\left[\alpha_{l_{1} l} \alpha_{l_{3} l}^{*} \alpha_{l_{3} l_{2}} \alpha_{l_{1} l_{2}}^{*}+(\alpha \rightarrow \beta)\right]\right\},
\end{aligned}
$$


and $w_{S}$ denotes the appropriate statistical factor $\left(w_{S}=1\right.$ for $l_{1} \neq l_{3}$ and $w_{S}=\frac{1}{2}$ for $\left.l_{1}=l_{3}\right)$. The present experimental bounds on these parameters are given in Table 10 .

\begin{tabular}{||c||c|c|c|c||}
\hline$l$ & $l_{1}^{-}$ & $l_{2}^{+}$ & $l_{3}^{-}$ & Bounds on $\mathcal{C}_{l_{1} l_{2} l_{3}}$ \\
\hline \hline$\mu$ & $e^{-}$ & $e^{+}$ & $e^{-}$ & $<1.0 \times 10^{-12}$ \\
\hline & $e^{-}$ & $e^{+}$ & $e^{-}$ & $<1.9 \times 10^{-5}$ \\
& $\mu^{-}$ & $\mu^{+}$ & $\mu^{-}$ & $<2.4 \times 10^{-5}$ \\
& $\mu^{-}$ & $e^{+}$ & $\mu^{-}$ & $<2.0 \times 10^{-5}$ \\
& $e^{-}$ & $\mu^{+}$ & $e^{-}$ & $<1.9 \times 10^{-5}$ \\
& $\mu^{-}$ & $\mu^{+}$ & $e^{-}$ & $<2.0 \times 10^{-5}$ \\
& $e^{-}$ & $e^{+}$ & $\mu^{-}$ & $<1.9 \times 10^{-5}$ \\
\hline
\end{tabular}

Table 10: 90\% CL limits 8, 40] on products of quartic polynomials in the couplings.

In general, the bounds in Tables 7 and 10 affect different combinations of the original couplings. The first gives us combined information on the charged and neutral lepton sectors, while the second constrains only the charged lepton sector. However, in many models, these couplings are related. As an example, let us assume that all diagonal couplings are of order one with all off-diagonal couplings suppressed. Then, from Table 7 we find

$$
\begin{aligned}
\left|\alpha_{e \mu}\right|^{2} & <3.7 \times 10^{-4} ; \quad\left|\beta_{e \mu}\right|^{2}<2.5 \times 10^{-2}, \\
\left|\alpha_{\mu \tau}\right|^{2} & <4.7 \times 10^{-2}, \\
\left|\alpha_{e \tau}\right|^{2} & <6.7 \times 10^{-2},
\end{aligned}
$$

while the bound on $l \rightarrow 3 l^{\prime}$ provides much more stringent limits,

$$
\begin{aligned}
\left|\alpha_{e \mu}\right|^{2}+\left|\beta_{e \mu}\right|^{2} & <3.3 \times 10^{-13}, \\
\left|\alpha_{\mu \tau}\right|^{2}+\left|\beta_{\mu \tau}\right|^{2} & <8.0 \times 10^{-6}, \\
\left|\alpha_{e \tau}\right|^{2}+\left|\beta_{e \tau}\right|^{2} & <6.3 \times 10^{-6} .
\end{aligned}
$$

On the other hand, if we keep the assumption that the diagonal couplings are dominant, but there is a hierarchy between the neutrino and lepton couplings of order

$$
\frac{\theta_{m m}}{\alpha_{l l}} \sim \mathcal{O}\left(10^{2}\right)
$$

then the information in the Michel parameters becomes comparable with that in $\tau \rightarrow 3 e$ and $\tau \rightarrow 3 \mu$ decays. Of course, this last case will only seem 'natural' in the presence of some (maybe approximate) symmetry suppressing the charged-lepton couplings to the new neutral vector.

\section{A.2 Neutral Scalar Bosons}

The interaction of a new flavour-violating, neutral scalar boson $S^{0}$ with the leptons can be written as

$$
\mathcal{L}=J S^{0},
$$


where

$$
J=\left(M_{S} \sqrt{\sqrt{2} G_{F}}\right)\left[\bar{l}\left(A^{\dagger} \gamma_{R}+A \gamma_{L}\right) l+\bar{\nu}\left(B^{\dagger} \gamma_{R}+B \gamma_{L}\right) \nu\right],
$$

$M_{S}$ is the mass of the neutral scalar, and $A$ and $B$ are $3 \times 3$ matrices in the respective flavour spaces. Again, since $M_{S}$ is typically much larger than the energy scale of $\mu$ and $\tau$ decays, the interaction is effectively of the four fermion type. After Fierzing, the $\bar{l}^{i} l^{j} \bar{\nu}^{m} \nu^{n}$ interaction is described by the hamiltonian (1), with effective couplings:

$$
\begin{array}{cl}
g_{l_{R}^{i} l_{L}^{j}}^{S}=2 g_{l_{R}^{i} l_{L}^{j}}^{T}=A_{i j} B_{m n}, & g_{l_{R}^{i} l_{L}^{j}}^{V}=A_{i j} B_{n m}^{*}, \\
g_{l_{L}^{i} l_{R}^{j}}^{S}=2 g_{l_{L}^{i} l_{R}^{j}}^{T}=A_{j i}^{*} B_{n m}^{*}, & g_{l_{L}^{i} l_{R}^{j}}^{V}=A_{j i}^{*} B_{m n} .
\end{array}
$$

The LL and RR couplings vanish. Notice the relation

$$
g_{l_{R}^{i} l_{L}^{j}}^{S} g_{l_{L}^{i} l_{R}^{j}}^{S}=g_{l_{R}^{i} l_{L}^{j}}^{V} g_{l_{L}^{i} l_{R}^{j}}^{V} .
$$

Since the neutrinos are unobserved, the measurable vector and scalar effective couplings are also related:

$$
\begin{gathered}
g_{l_{R}^{\prime} l_{L}}^{V}=g_{l_{R}^{\prime} l_{L}}^{S}=2 g_{l_{R}^{\prime} l_{L}}^{T}=A_{l^{\prime} l} \Omega, \\
g_{l_{L}^{\prime} l_{R}}^{V}=g_{l_{L}^{\prime} l_{R}}^{S}=2 g_{l_{L}^{\prime} l_{R}}^{T}=A_{l l^{\prime}}^{*} \Omega,
\end{gathered}
$$

where

$$
\Omega=\left[\sum_{m, n}\left|B_{m n}\right|^{2}\right]^{1 / 2} .
$$

This new flavour-changing neutral scalar also contributes to the decays $l^{-} \rightarrow l_{1}^{-} l_{2}^{+} l_{3}^{-}$. The corresponding decay widths are given by

$$
\Gamma\left[l^{-} \rightarrow l_{1}^{-} l_{2}^{+} l_{3}^{-}\right]=\frac{m_{l}^{5} G_{F}^{2}}{192 \pi^{3}} \mathcal{D}_{l_{1} l_{2} l_{3}}^{l},
$$

where the $\mathcal{D}_{l_{1} l_{2} l_{3}}^{l}$ are quartic polynomials in the charged-lepton couplings:

$$
\begin{aligned}
\mathcal{D}_{l_{1} l_{2} l_{3}}^{l}=w_{S} & \left\{\left(\left|A_{l_{1} l}\right|^{2}+\left|A_{l l_{1}}\right|^{2}\right)\left(\left|A_{l_{2} l_{3}}\right|^{2}+\left|A_{l_{3} l_{2}}\right|^{2}\right)\right. \\
& +\left(\left|A_{l_{3} l}\right|^{2}+\left|A_{l l_{3}}\right|^{2}\right)\left(\left|A_{l_{1} l_{2}}\right|^{2}+\left|A_{l_{2} l_{1}}\right|^{2}\right) \\
& \left.-\operatorname{Re}\left[A_{l_{1} l} A_{l_{3} l}^{*} A_{l_{3} l_{2}} A_{l_{1} l_{2}}^{*}+A_{l l_{1}}^{*} A_{l l_{3}} A_{l_{2} l_{3}}^{*} A_{l_{2} l_{1}}\right]\right\} .
\end{aligned}
$$

For the case of $\mu \rightarrow 3 e$, this combination of couplings is greatly simplified,

$$
\mathcal{D}_{e e e}^{\mu}=\frac{3}{2}\left(\left|A_{\mu e}\right|^{2}+\left|A_{e \mu}\right|^{2}\right)\left|A_{e e}\right|^{2} .
$$

Since we have taken identical normalizations, the experimental bounds on these parameters are the same as those in Table 10.

Again, unless in the specific model one is studying some of the couplings to the charged leptons are suppressed with respect to the neutrino couplings, the bounds from the decays $l^{-} \rightarrow l_{1}^{-} l_{2}^{+} l_{3}^{-}$will be much stronger than those from $l^{-} \rightarrow l^{\prime} \nu \bar{\nu}$. 


\section{References}

[1] ALEPH Collab. , D. Buskulic et al, Phys. Lett. B346, 379 (1995).

[2] ARGUS Collab. , H. Albrecht et al, Phys. Lett. B341, 441 (1995).

[3] ARGUS Collab. , H. Albrecht et al, DESY 95-011.

[4] F. Scheck, Leptons, Hadrons and Nuclei (North-Holland, Amsterdam, 1983); Phys. Rep. 44, 187 (1978).

[5] C. Bouchiat and L. Michel, Phys. Rev. 106, 107 (1957).

[6] T. Kinoshita and A. Sirlin, Phys. Rev. 107, 593 (1957); 108, 844 (1957).

[7] L. Michel, Proc. Phys. Soc. A63, 514 (1950); ibid. A63, 1371 (1950).

[8] Particle Data Group, L. Montanet et al., Phys. Rev. D 50, 1173 (1994).

[9] K. Mursula and F. Scheck, Nucl. Phys. B253, 189 (1985);

K. Mursula, M. Roos and F. Scheck, Nucl. Phys. B219, 321 (1983).

[10] W. Fetscher, H.-J. Gerber and K. F. Johnson, Phys. Lett. B173, 102 (1986).

[11] W.J. Marciano and A. Sirlin, Phys. Rev. Lett. 61, 1815 (1988).

[12] ALEPH Collab. , D. Buskulic et al, CERN-PPE/95-03, 1995.

[13] C. Jarlskog, Nucl. Phys. 75, 659 (1966).

[14] Y.S. Tsai, Phys. Rev. D4, 2821 (1971).

[15] S. Kawasaki, T. Shirafuji and S.Y. Tsai, Progr. Theor. Phys. 49, 1656 (1973).

[16] S.-Y. Pi and A.I. Sanda, Ann. Phys. (NY) 106, 171 (1977).

[17] J.J. Gómez-Cadenas, Beautiful $\tau$ Physics in the Charm Land, in Proc. Tau-Charm Factory Workshop (SLAC, 1989), ed. L.V. Beers, SLAC-Report-343, p. 48 (1989).

[18] C.A. Nelson, Phys. Rev. D43, 1465 (1991); Phys. Rev. Lett. 62, 1347 (1989); Phys. Rev. D40, 123 (1989) [Err: D41, 2327 (1990)].

[19] S. Goozovat and C.A. Nelson, Phys. Rev. D44, 2818 (1991); Phys. Lett. B267, 128 (1991) [Err: B271, 468 (1991)].

[20] W. Fetscher, Phys. Rev. D 42, 1544 (1990).

[21] J. Bernabéu, A. Pich and N. Rius, Phys. Lett. B257, 219 (1991).

[22] R. Alemany et al, Nucl. Phys. B379, 3 (1992).

[23] M. Davier et al, Phys. Lett. B306, 411 (1993). 
[24] ARGUS Collab. , H. Albrecht et. al, Phys. Lett. B316, 608 (1993).

[25] M. Davier, contribution to ref. [28]; J. Korolko, ibid; J. Raab, ibid; M. Schmidtler, ibid;

A. Rougé, Ecole Polytechnique preprint X-LPNHE 95-01.

[26] P. Langacker and D. London, Phys. Rev. D 39, 266 (1989).

[27] A. Santamaría, J. Bernabéu and A. Pich, Phys. Rev. D 36, 1408 (1987); ibid 32, 2461 (1985);

B. Grinstein, J. Preskill and M.B. Wise, Phys. Lett. B159, 57 (1985)

[28] Proc. Third Workshop on Tau Lepton Physics (Montreux, 1994), ed. L. Rolandi, Nucl. Phys. B (Proc. Suppl.) (to appear)

[29] W. Fetscher and H.-J. Gerber, in Precision Tests of the Standard Model, ed. P. Langacker, (World Scientific, Singapore, 1993).

[30] A. Stahl, Phys. Lett. B324, 121 (1994).

[31] A. Barroso and J. P. Silva, Phys. Rev. D 50, 4581 (1994).

[32] D.I. Britton et al, Phys. Rev. Lett. 68, 3000 (1992).

[33] G. Czapek et al, Phys. Rev. Lett. 70, 17 (1993).

[34] W.J. Marciano and A. Sirlin, Phys. Rev. Lett. 71, 3629 (1993).

[35] R. Decker and M. Finkemeier, Nucl. Phys. B438, 17 (1995); contribution to Ref. [28].

[36] UA1 Collaboration, C. Albajar et al, Z. Phys. C44, 15 (1989); Phys. Lett. B185, 233 (1987).

[37] UA2 Collaboration, J. Alitti et al, Z. Phys. C52, 209 (1991).

[38] CDF Collaboration, F. Abe et al, Phys. Rev. Lett. 68, 3398 (1992).

[39] W.J. Marciano, Tau Physics - A Theoretical Perspective, in Ref. 28].

[40] CLEO Collab. ,J. Bartelt et al, Phys. Rev. Lett. 73 (1994) 1890. 